\title{
The Shape of Stress Relaxation Curves and the Mechanical Equation of State in Molybdenum*
}

\author{
By Koji Tanoue**, Osamu Nakano*** and Hidehiko Matsuda**
}

\begin{abstract}
It has been demonstrated that a family of $\log \dot{\varepsilon}$ vs $\log \sigma$ curves, obtained from stress relaxation tests after monotonic loading, can be superposed on one another by translation (scaling) along a particular direction and that the locus of the translation is linear. The present study deals with the effects of (1) cyclic loading, (2) imposed strain rates, (3) impurities and (4) grain sizes on the shape of $\log \dot{\varepsilon}$ vs $\log \sigma$ curves and the scaling parameter, $m\left[=(\delta \ln \dot{\varepsilon} / \delta \ln \sigma)_{\lambda}\right]$ defined as a reciprocal of the slope of the locus in scaling. Stress relaxation curves are concave, and the linear relationship of the locus holds for scaling except for the stress relaxation after fatigue tests and that of single crystals. Measured $m$ values are dependent on impurities and temperature, and independent of other factors. The physical meaning of the scaling locus is discussed referring to the experimental results. The locus of translation is explained by the relation between the preexponential term $\dot{\varepsilon}_{0}$ and the maximum internal stress $\sigma_{i}^{0}$. The mechanical equation of state, $f\left(\dot{\varepsilon}, \sigma,\left(\dot{\varepsilon}_{0}, \sigma_{i}^{0}\right), T\right)=0$ is shown to be valid for an arbitrary value of $(\dot{\varepsilon}, \sigma, T)$, provided the scaling is possible.
\end{abstract}

(Received December 2, 1980)

Keyzords : mechanical equation, stress relaxation, low temperature deformation, molybdenum

\section{Introduction}

Stress relaxation tests give a unique relationship between the flow stress $\sigma$ and the plastic strain rate $\dot{\varepsilon}$ with the almost constant microstructure, because the plastic strain during the tests is very small. Stress relaxation (or constant hardness) curves, plotted as $\log \sigma$ vs $\log \dot{\varepsilon}$, change their shape depending on the temperature and the history of prestrain. It is known that the shape is concave upward at low temperatures $\left(T<0.25 T_{\mathrm{m}} ; T_{\mathrm{m}}\right.$ a melting point), while it is concave downward at high temperatures $\left(T>0.25 T_{\mathrm{m}}\right)$. The curvature and slope decrease with increasing strain at low temperatures. Contrary to this, they increase as the plastic strain increases at high temperatures ${ }^{(1)}$. Experimental results also show that stress relaxation curves for polycrystalline materials can be superposed on one another by translation (scaling) in a particular direction, and

* This paper was originally published in Japanese in J. Japan Inst. Metals, 45 (1981), 566.

** Department of Metal Processing Engineering, Kyushu Institute of Technology, Kitakyushu 804, Japan.

*** Graduate Student, Kyushu Institute of Technology. Present address: Nippon Tungsten Co., Ltd., Fukuoka 815, Japan. that a linear relationship holds for scaling. The scaling parameter $m(=1 / \mu)$ obtained as a reciprocal of the slope on scaling agrees with the exponent of the power law for high temperature creep in aluminium ${ }^{(1)-(4)}$. Thus, the scaling relation is thought to be a locus between $\sigma$ and $\dot{\varepsilon}$ of steady state creep at high temperature deformation.

There are several reports on the theoretical interpretation of scaling relation at low temperature deformation. Hart ${ }^{(5)}$ has assumed that the plastic strain rate during stress relaxation is given by Gilman's empirical equation ${ }^{(6)}$ as follows:

$$
\dot{\varepsilon}=\alpha \rho_{\mathrm{m}} v_{1} \exp \left(-\sigma_{0} / \sigma\right),
$$

where $\alpha$ is the geometrical factor, $\rho_{\mathrm{m}}$ the mobile dislocation density, $v_{1}$ the frequency factor, $\sigma_{0}$ a parameter dependent on the structure. Supposing that $\rho_{\mathrm{m}}, v_{1}$ and $\sigma_{0}$ are constant during tests, eq. (1) gives

$$
\begin{aligned}
(\partial \ln \dot{\varepsilon} / \partial \ln \sigma)_{e} & =\sigma_{0} / \sigma \\
& =1 / \lambda,
\end{aligned}
$$

where $\lambda\left[=(\partial \ln \sigma / \partial \ln \dot{\varepsilon})_{\varepsilon}\right]$ is the strain rate sensitivity parameter obtained as a slope of the stress relaxation curves. If a linear scaling relation holds among the curves, the following equation must be satisfied. 


$$
\mu=1 / m=(\delta \ln \sigma / \delta \ln \dot{\varepsilon})_{\lambda},
$$

where $\delta$ signifies a small change in $\ln \sigma$ and $\ln \dot{\varepsilon}$ on the scaling line. Combination of eqs. (1), (2) and (3) give

$$
m=\operatorname{dln} \rho_{\mathrm{m}} / \mathrm{d} \ln \sigma_{0} .
$$

On the other hand, the authors asserted the following Johnston-Gilman type equation to be valid for stress relaxation phenomena and attempted to explain the scaling relation ${ }^{(7)}$ :

$$
\dot{\varepsilon}=\alpha \rho_{\mathrm{m}} b B \sigma^{* \mathrm{~m}^{*}},
$$

where $b$ is the Burgers vector, $B$ a parameter dependent on the temperature, $\sigma^{*}$ the effective stress, $m^{*}$ the dislocation velocity-stress exponent. The shape of relaxation run and its scaling can, respectively, be described by the following phenomenologically derived equations $^{(1)(7)}$.

$$
\begin{aligned}
\ln \left(\sigma / \sigma_{\mathrm{c}}\right) & =\exp \left[\omega \ln \left(\dot{\varepsilon} / \dot{\varepsilon}_{\mathrm{c}}\right)\right] \\
& =\left(\dot{\varepsilon} / \dot{\varepsilon}_{\mathrm{c}}\right)^{\omega}
\end{aligned}
$$

and

$$
\dot{\varepsilon}_{\mathrm{c}}=A \sigma_{\mathrm{c}}^{\mathrm{m}},
$$

where $\sigma_{\mathrm{c}}$ and $\dot{\varepsilon}_{\mathrm{c}}$ are, respectively, the stress and the strain rate when the deformation stagnates, and $\omega$ the parameter concerning the shape of relaxation run, $A$ the constant deciding the scaling position. As the condition of $0<\sigma^{*} /$ $\sigma_{\mathrm{c}} \leqq 1$ was satisfied at the low temperature deformation in molybdenum, $m$ was obtained from eqs. (5)-(7) as a first approximation to be (see ref. (7) for further details)

$$
m=m^{*}+\operatorname{d} \ln \rho_{\mathrm{m}} / \mathrm{d} \ln \sigma_{\mathrm{c}} .
$$

Equation (1), on which eq. (4) was based, has not so much wide application as eq. (5) to the stress relaxation phenomenon as reported in much literature. Therefore, it is not clear whether eq. (4) can explain the practical scaling relation at the low temperature deformation. Equation (8) is also the one derived from a rough approximation. Thus, neither eq. (4) nor eq. (8) gives the satisfactory solution to the physical meaning of scaling at the low temperature deformation.

The purpose of the present paper is to investigate the effects of various factors such as loading mode, strain rate, impurity, grain size and temperature on the scaling parameter $m$, and clarify the physical meaning of scaling and the condition under which the mechanical equation of state holds.

\section{Experimental Procedure}

Materials used in this experiment are sintered pure molybdenum wires $(\phi 1.3 \mathrm{~mm})$ and plates (1 mm thickness) of commercial grade (above $99.95 \%$ ), and high purity molybdenum ingots (above $99.99 \%$ ) prepared from Johnson-Matthey Ltd. Table 1 shows the chemical composition of the specimens. The wires and the plates were mechanically shaped to be $\phi 1.2 \mathrm{~mm} \times$ $50 \mathrm{~mm}$ and $1 \mathrm{~mm} \times 3 \mathrm{~mm} \times 50 \mathrm{~mm}$, respectively, and their surface were electropolished. The specimens were then heat treated to arrange the grain size in a vacuum of $2 \times 10^{-4} \mathrm{~Pa}$. The heat treatment for $3 \mathrm{ks}(50 \mathrm{~min})$ at $1673 \mathrm{~K}$ produced the mean grain size of $23 \mu \mathrm{m}$ for the wires. On the other hand, the nominal grain sizes of $30,50,100$ and $1500 \mu \mathrm{m}$ were prepared for the plates by a double strain annealing procedure which was reported in a previous paper $^{(8)}$. Single crystals were grown by the electron beam vertical floating zone melting of high purity molybdenum ingots. The specimens of rectangular prism with a base of $2 \mathrm{~mm} \times$ $2 \mathrm{~mm}$ and a height of $6 \mathrm{~mm}$ were prepared for compression test by spark cutting of the grown crystals so that the compressive axis may agree

Table 1 Chemical composition of specimens ( $\mathrm{ppm}$ in weight).

\begin{tabular}{lrrrrrrrrr}
\hline \multicolumn{1}{c}{ Materials } & $\mathrm{Fe}$ & $\mathrm{Ni}$ & $\mathrm{Cu}$ & $\mathrm{Co}$ & $\mathrm{Sn}$ & $\mathrm{O}$ & $\mathrm{N}$ & $\mathrm{C}$ & NVR* \\
\hline Pure Mo (wire) & 20 & 10 & $<30$ & - & - & 26 & 8 & $<30$ & 10 \\
Pure Mo (plate) & 80 & $<30$ & $<30$ & - & - & 160 & 7 & $<30$ & 40 \\
Mo-Co alloy & 20 & 10 & - & 510 & - & - & - & - & 10 \\
Pure Mo (Johnson-Matthey) & 5 & 3 & 3 & - & 30 & - & - & - & 25 \\
\hline \hline
\end{tabular}

* Non volatilized residuum containing $\mathrm{Al}, \mathrm{Ca}, \mathrm{Si}$ and $\mathrm{Mg}$. 
with [149] direction. The specimens of single crystals were then annealed for $18 \mathrm{ks}(5 \mathrm{~h})$ at $1873 \mathrm{~K}$ in vacuum after electropolishing.

The wire and plate specimens with a gage length of $25 \mathrm{~mm}$ were deformed in tension at the nominal plastic strain rate of $\dot{\varepsilon}_{1}=3.3 \times$ $10^{-4} \mathrm{~s}^{-1}$. The temperatures were 292 (300 in the plates), 350, 420 (400 and 450 in the plates), 500 and $550 \mathrm{~K}$. The compression tests were made for the single crystals using a lubricant made of fine graphite powders at the nominal plastic shear strain rate of $\dot{\gamma}_{\mathrm{a}}=4.8 \times 10^{-4} \mathrm{~s}^{-1}$ and $300 \mathrm{~K}$. The relaxed stress rate $(-\dot{\sigma})$ was calculated from the Lagrange interpolation method with 50 data points on a load relaxation curve. The plastic strain rate $\dot{\varepsilon}_{\mathrm{p}}$ is then obtained from $\dot{\varepsilon}_{\mathrm{p}}=-\dot{\sigma} / K_{\mathrm{c}}$ with a combined machine stiffness $K_{\mathrm{c}}$ as a function of the flow stress. Thus, one can get the stress relaxation curves plotted as $\log \sigma$ vs $\log \dot{\varepsilon}$.

In order to confirm again whether or not a series of stress relaxation curves forms one unique master curve by scaling and the scaling locus is linear, the scaling relation obtained by a monotonic loading mode was investigated in detail. Furthermore, it was also investigated how the scaling parameter $m(=1 / \mu)$ was influenced by various factors. In the present study, (1) cyclic loading, (2) imposed strain rate (shown in Fig. 1), (3) impurities and (4) grain size were selected as the factors. In (1) cyclic loading (A), stress relaxation tests were made after loading monotonically at $\dot{\varepsilon}_{1}$ to each strain and loading cyclically 2 to 500 times between 30 and 200 (or 400) MPa. When the number of cycles was less than 20 times, one relaxation run was obtained using a piece of specimen deformed to each strain after cyclic loading to a given cycle. On the other hand, a single specimen was repeatedly employed when the number of cycles was more than 50 times. Thus, the effect of the number of cycles and the stress amplitudes on $m$ were investigated. Figure 1-(1)(B) illustrates the fatigue test in a tension-tension mode prior to stress relaxation tests. The test was made at each cycle of $2 \times 10^{2}, 5 \times 10^{2}, 10^{4}$ and $10^{5}$ with the frequency of $30 \mathrm{~Hz}$ between 30 and $200 \mathrm{MPa}$. Figure 1-(2) shows the definition of the imposed strain rate $\dot{\varepsilon}_{2}$ changed variously from $10^{-5}$ to $10^{-3} \mathrm{~s}^{-1}$. In order to know the effect of (3)

\section{(I) Cyclic Loading}
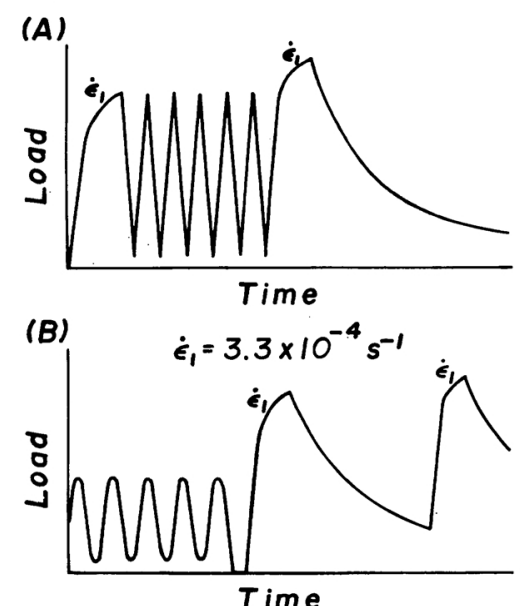

(2) Imposed Strain Rate

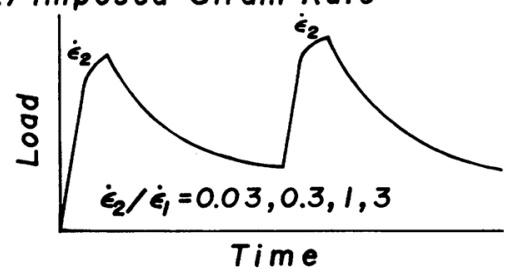

Fig. 1 Schematic representations of (1) cyclic loading and (2) imposed strain rate as various factors to investigate their effects on the shape of stress relaxation curves plotted as $\log \sigma$ vs $\log \dot{\varepsilon}$ and the stressstrain rate scaling parameter, $m\left\{=(\delta \ln \dot{\varepsilon} / \delta \ln \sigma)_{\lambda}\right\}$.

impurities on $m$, three materials (the wires, the plates and Mo-0.1 at \% Co alloy given in Table 1) were prepared. The scaling parameter $m$ in these materials was determined from the relaxation curves following monotonic loading. The effect of (4) grain size on $m$ was also investigated using four nominal grain sizes of 30 , 50, 100 and $1500 \mu \mathrm{m}$. Single crystals with the (101)[111] slip system were tested by compression to know about the shape of stress relaxation curves and their scaling relation. The stress relaxation curve for single crystals is expressed as log-log plots of the shear stress $\tau$ and the shear plastic strain rate $\dot{\gamma}_{\mathrm{p}}$. They were calculated from the following equations.

$$
\tau=\frac{P \sin \chi_{0}}{a_{0}(1-e)} \sqrt{(1-e)^{2}-\sin ^{2} \lambda_{0}}
$$

and

$$
\dot{\gamma}_{\mathrm{p}}=\frac{-\dot{\sigma}}{K_{\mathrm{c}}}\left[\frac{(1-\varepsilon)}{\sin \chi_{0} \sqrt{(1-\varepsilon)^{2}-\sin ^{2} \lambda_{0}}}\right],
$$


where $P$ is a load, $a_{0}$ the initial cross-sectional area of specimen, $\dot{\sigma}$ the relaxed stress rate, $e$ the engineering strain, $\chi_{0}$ and $\lambda_{0}$ the angle between the compressive axis and the slip plane, the slip direction, respectively. The relation between $e$, $\varepsilon$ and $\sigma$ is related by

$$
\begin{aligned}
& e=\left(l_{\mathrm{c}}-\frac{\dot{P}}{S_{\mathrm{m}}}\right) / l_{0}, \\
& \varepsilon=\frac{l_{\mathrm{c}}}{l_{0}}-\frac{\sigma}{K_{\mathrm{c}}},
\end{aligned}
$$

and

$$
\sigma=P(1-e) / a_{0},
$$

where $S_{\mathrm{m}}$ is the machine stiffness, $l_{\mathrm{c}}$ the crosshead displacement. Thus, the shear stress $\tau$ and the plastic shear strain rate $\dot{\gamma}_{p}$ during stress relaxation can be obtained from eqs. (9)-(13) as a function of $P$ and $\dot{P}$.

\section{Experimental Results}

Figure 2 shows a series of stress relaxation curves following monotonic loading for molybdenum wires at $292 \mathrm{~K}$. The plastic strain rates at the beginning of relaxation agreed with the imposed strain rate $\dot{\varepsilon}_{1}$. These curves can be superposed by translating in the direction of the oblique line and form a unique master curve.

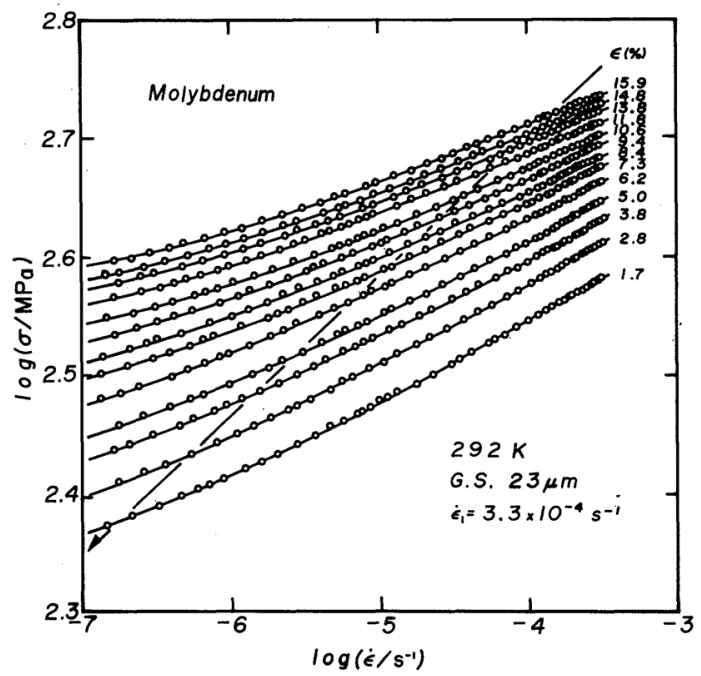

Fig. 2 A series of stress relaxation curves following monotonic loading for molybdenum at $292 \mathrm{~K}$. The diagonal straight line is the direction of scaling.
The practical scaling relation is given in Fig. 3. Each point of data indicates the coordinate on each relaxation curve which can be superposed on one point arbitrarily selected on the curve with $1.7 \%$ strain. There exists obviously a good linear relationship with the slope of 0.12 $(m=8.6)$.

Figure 4 represents the result by cyclic loading (A), taking 2 cycles as an example. A linear relationship holds again on scaling. The master curve formed by translating the curves in Fig. 4 onto the curve with $2.4 \%$ strain is given in Fig. 5. The similar shape was obtained for the other number of cycles less than 500 times. A typical example for cyclic loading (B) is shown in Fig. 6 . The strain rate sensitivity parameter $\lambda$ remains constant during each relaxation run, however, $\lambda$ decreases with increasing plastic strain prior to stress relaxation. Therefore, these curves could not be superposed on each

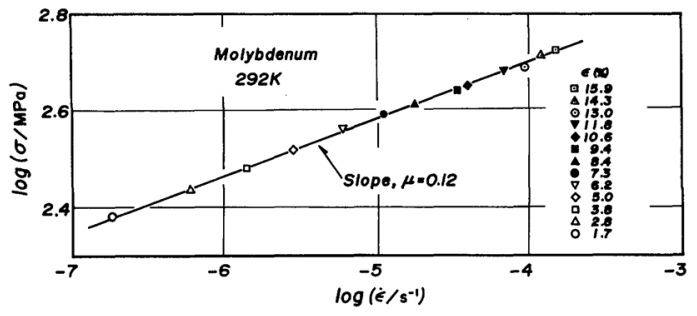

Fig. 3 The scaling relation for the stress relaxation curves shown in Fig. 2.

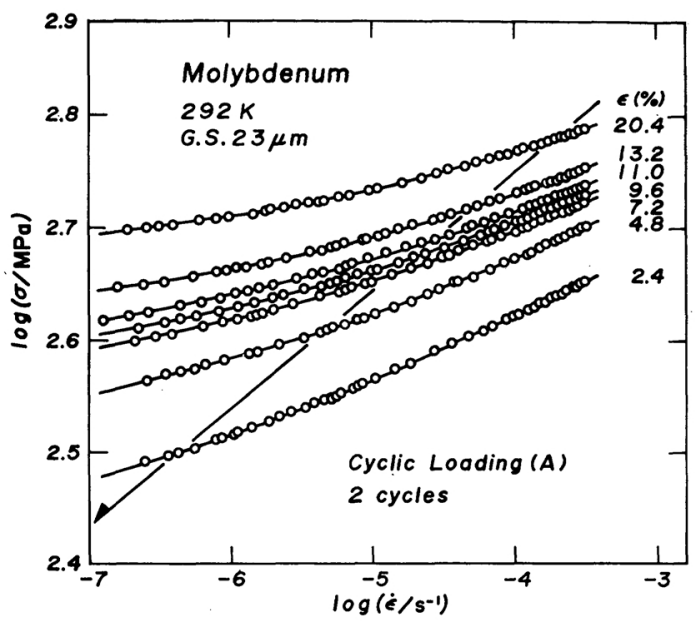

Fig. 4 A series of stress relaxation curves following cyclic loading (A) of 2 cycles for molybdenum at $292 \mathrm{~K}$. 


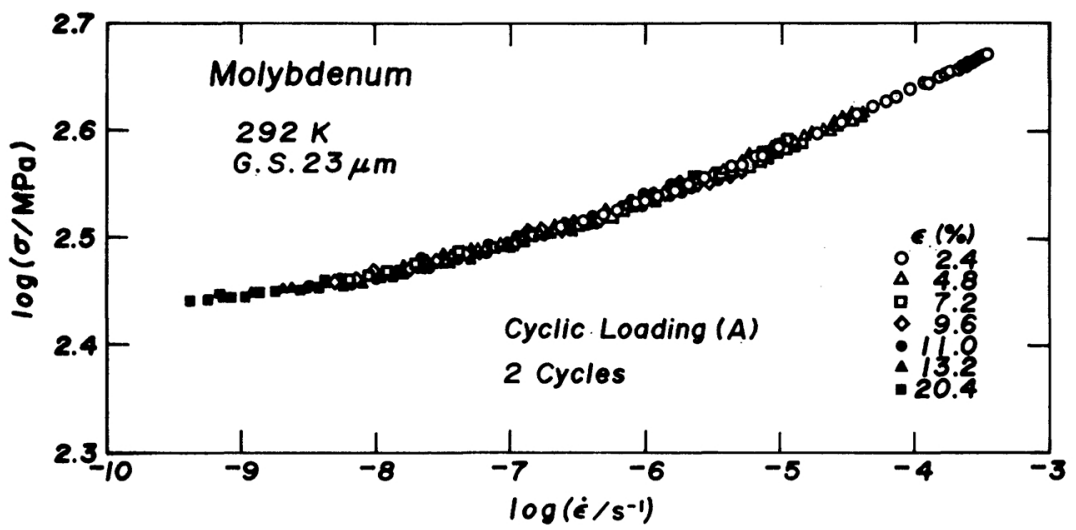

Fig. 5 Master curve generated from seven curves following cyclic loading (A) of 2 cycles at $292 \mathrm{~K}$. It is constructed by superposing curves in Fig. 4 onto the curve with $2.4 \% \varepsilon$.

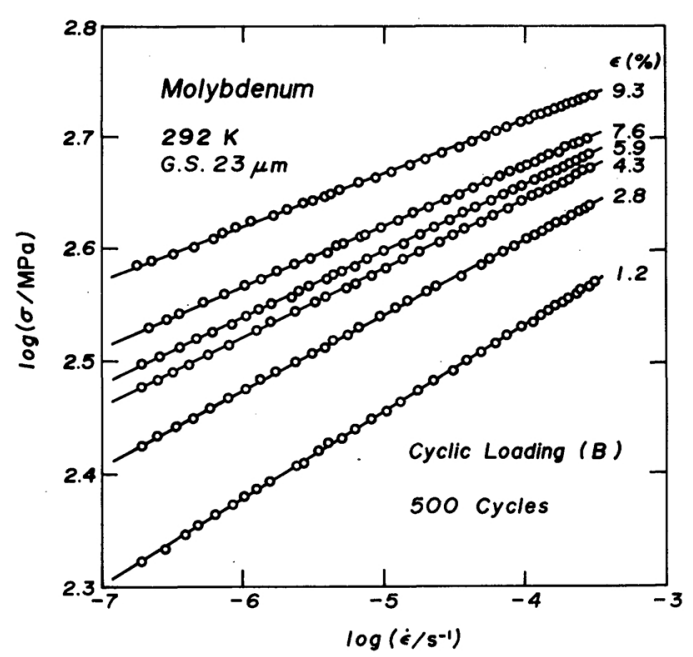

Fig. 6 A series of stress relaxation curves following cyclic loading (B) of 500 cycles for molybdenum at $292 \mathrm{~K}$.

other by scaling. The similar results were obtained for the other number of cycles 200 to $10^{5}$. Figure 7 shows the result at (2) the imposed strain rate of $10^{-5} \mathrm{~s}^{-1}$. The initial strain rate of stress relaxation agreed with the imposed strain rate $\dot{\varepsilon}_{2}$, and the linear relationship held on scaling. The scaling parameter $m$ was to be 8.6 independent of $\dot{\varepsilon}_{2}$. The effect of (3) impurities on $m$ was investigated using Mo-Co alloy besides pure molybdenum. The results are given in Fig. 8. It is known from the figure that $\lambda$ is small compared with that of pure molybdenum wires. The effect of various factors on $m$ are summarized in Fig. 9. The

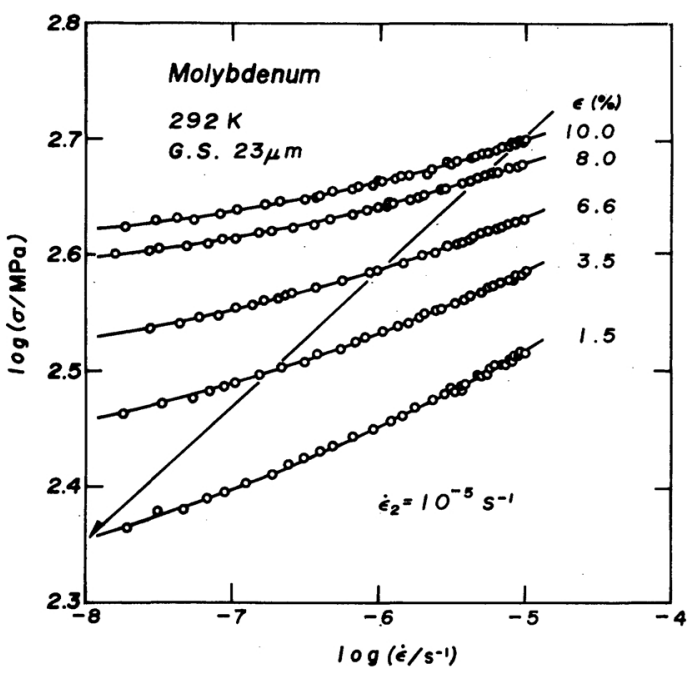

Fig. 7 A series of stress relaxation curves tested at the imposed strain rate of $\dot{\varepsilon}_{2}=10^{-5} \mathrm{~s}^{-1}$ for molybdenum at $292 \mathrm{~K}$.

results of the pure molybdenum plates are shown in Fig. 11. Though the number of cycles, the stress amplitude in cyclic loading and the imposed strain rate did not alter the magnitude of $m$, the impurities lowered $m$ considerably, as seen in Mo-Co alloy. The effect of (4) grain size on $m$ is presented in Fig. 10. The parameter $m$ was independent of grain size. Several specimens with $1500 \mu \mathrm{m}$ grain size exhibited severe inhomogeneous deformation. They failed to show a linear scaling relationship. Figure 11 shows that the mean $m$ values become more insensitive to temperature in the order of wires, 


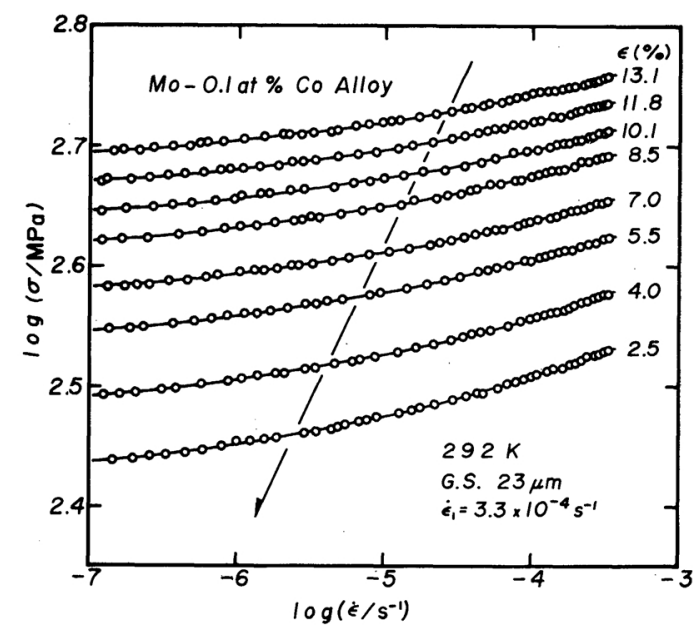

Fig. 8 A series of stress relaxation curves for Mo0.1 at $\%$ Co alloy at $292 \mathrm{~K}$.

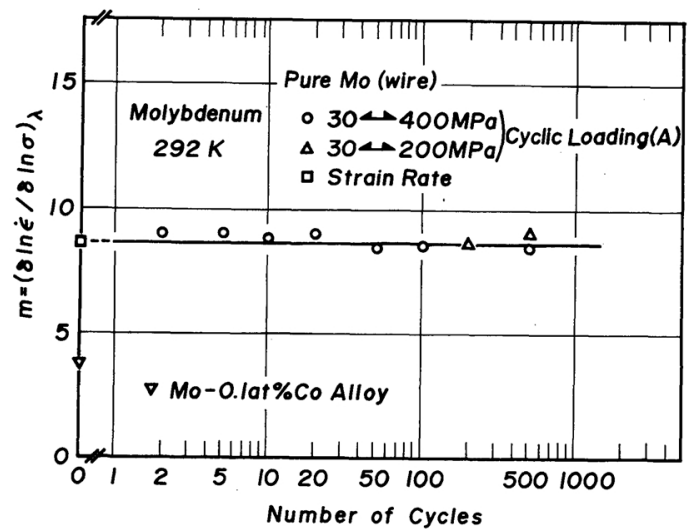

Fig. 9 The effects of cyclic loading (A), imposed strain rate and impurities on $m$ at $292 \mathrm{~K}$.

plates and Mo-Co alloy. It is known from Table 1 that the decrease in $m$ like this may result from the increase in impurities.

Several single crystals with the (101)[111] slip system were simply compressed at the nominal plastic shear strain rate $\dot{\gamma}_{\mathrm{a}}$ of $4.8 \times$ $10^{-4} \mathrm{~s}^{-1}$ and the testing temperature of $300 \mathrm{~K}$. The other specimens were used for stress relaxation tests under the same condition. A typical shear stress-plastic shear strain curve is given in Fig. 12. The arrows in the figure indicate the strain at which the stress relaxations were performed. Figure 13 shows the resultant relaxation runs. They were concave downward, and $\lambda$ increased continuously, as the applied

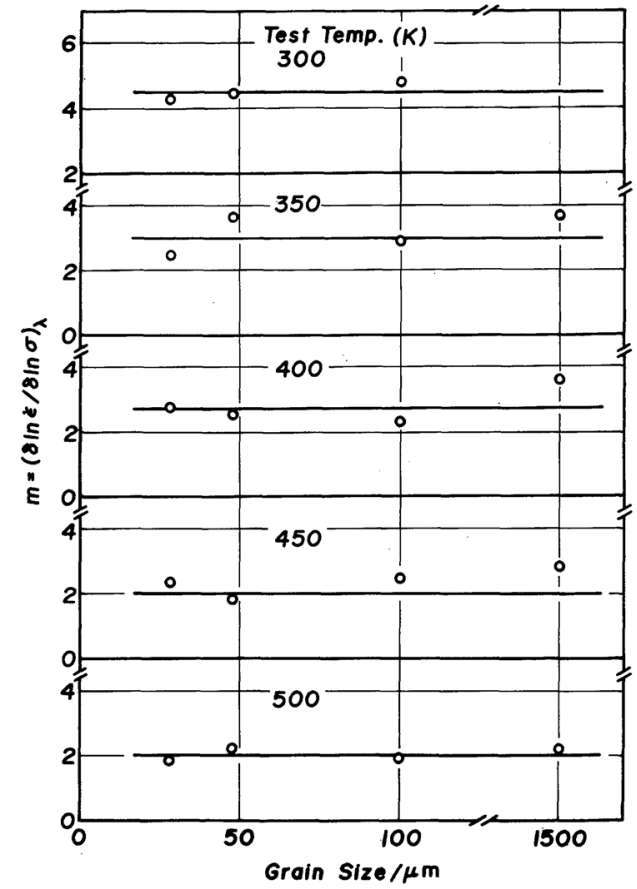

Fig. 10 The effect of grain size on $m$ at various temperatures.

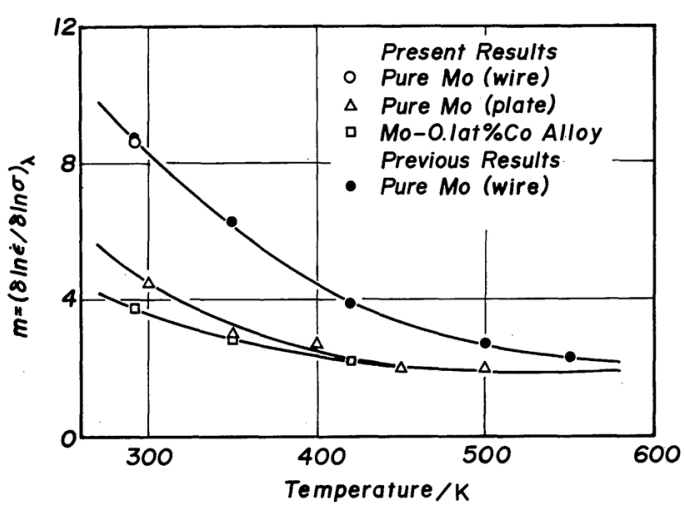

Fig. 11 Variations of $m$ with temperature for several materials.

stress decreased. The dotted line in the vertical direction signifies the true plastic shear strain rate $\dot{\gamma}_{p_{0}}\left[=\dot{\gamma}_{\mathrm{a}}\left(1-\mathrm{d} \tau / \mathrm{d} \gamma \cdot 1 / 4 K_{\mathrm{c}}\right)\right]$ calculated from the curve in Fig. 12. It approaches the nominal strain rate $\dot{\gamma}_{\mathrm{a}}$, as the strain increases, namely as $\mathrm{d} \tau / \mathrm{d} \gamma$ becomes zero. The fact that the initial strain rates at the beginning of relaxation run did not always agree with $\dot{\gamma}_{p_{0}}$ may be due to the very small combined machine stiffness $K_{c}$ 


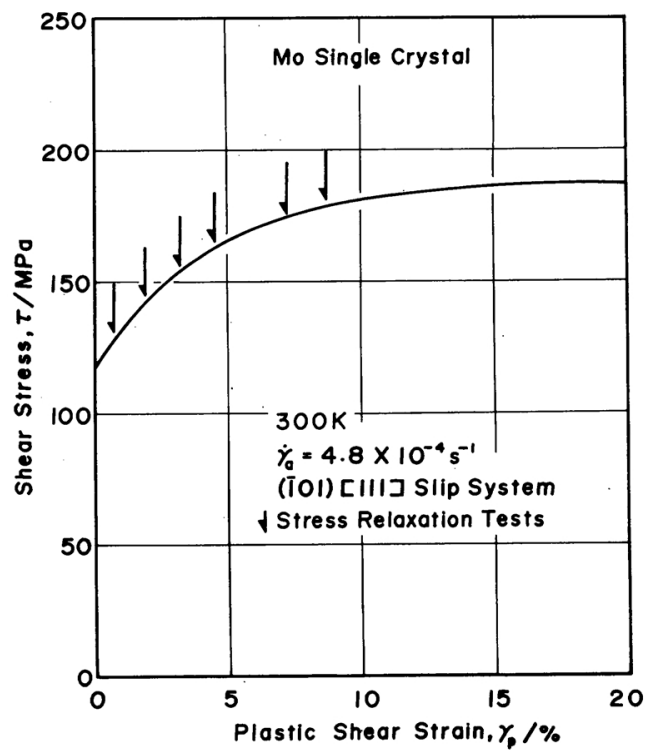

Fig. 12 Shear stress vs plastic shear strain curve for a molybdenum single crystal with (101)〈111〉 slip system tested by compression at $300 \mathrm{~K}$. The apparent shear strain rate, $\dot{\gamma}_{\mathrm{a}}$ is $4.8 \times 10^{-4} \mathrm{~s}^{-1}$ and the arrows in the figure indicate the places where stress relaxation tests were performed.

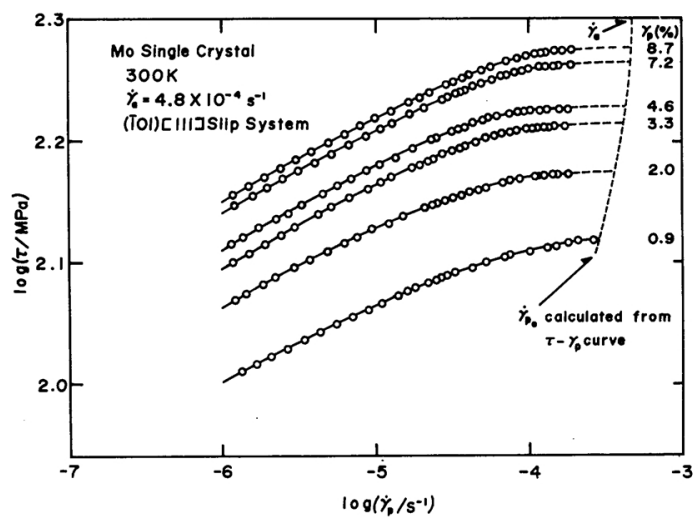

Fig. 13 A series of stress relaxation curves plotted as $\log \tau$ vs $\log \dot{\gamma}_{\mathrm{p}}$ for molybdenum single crystals with (101) [111] slip system at $300 \mathrm{~K}$.

(=7-9 GN/m $\left.\mathrm{m}^{2}\right)$ in compression compared with $K_{\mathrm{c}}\left(=20-50 \mathrm{GN} / \mathrm{m}^{2}\right)$ in tension ${ }^{(8)}$. After all the scaling relation did not hold among the curves for single crystals. The plastic strain during stress relaxation was less than $0.2 \%$ for polycrystals and about $2 \%$ (in shear strain) for single crystals.

\section{Discussion}

\section{The shape of stress relaxation curves}

It is known from Figs. 2 and 3 that the stress relaxation curves obtained by monotonic loading are concave upward and the linear scaling relationship holds. The shape of these curves was not influenced by such factors as (1)-(A) the number of cycles and the amplitude of cyclic loading and (2) the imposed strain rate as shown in Fig. 1. This result can be explained in the following way. The dislocation velocity-stress exponent $m^{*}$ is derived from eqs. (2) and (5) under the condition of $\mathrm{d} \sigma=\mathrm{d} \sigma^{*}$ to be

$$
m^{*}=\left(1-\frac{\sigma_{\mathbf{i}}}{\sigma}\right) / \lambda \text {. }
$$

Equation (14) means that $\lambda$ increases as $\sigma$ increases provided $m^{*}$ and the internal stress ${ }^{\dagger} \sigma_{\mathrm{i}}$ are constant, namely the stress relaxation curves become concave upward. The increase in the number of cycles in case of (1)-(A) may have little effect on the microstructure or $\sigma_{i}$, because most of the strains during cyclic loading are elastic. The imposed strain rate has also no effect on $\sigma_{\mathrm{i}}$ but changes the effective stress $\sigma^{*}$ only. In other words, such factors as (1)-(A) and (2) can not contribute to the increase in $\sigma_{\mathbf{i}}$ which is solely caused by the increase of plastic strain induced by monotonic loading. Accordingly, the microstructure or $\sigma_{i}$ during stress relaxation remains almost constant even if a specimen has experienced such a deformation history as (1)-(A) and (2) shown in Fig. 1. On the other hand, the straight relaxation curves with different $\lambda$ (see Fig. 6) were obtained after the fatigue tests given in (1)-(B), Fig. 1. These results can not be explained from eq. (14) under the condition that the equation holds, i.e. $\mathrm{d} \sigma=\mathrm{d} \sigma^{*}$ which implies $\sigma_{\mathrm{i}}$ independent of the applied stress. As $m^{*}$ is the parameter de-

$\dagger$ The internal stress $\sigma_{1}$ is used as the mean internal stress $\sigma_{1}^{*}$ directly connected to the mean velocity of dislocations in this equation. Though $\sigma_{1}^{*}$ is thought to be dependent on the applied stress, one can regard $\sigma_{i}^{*}$ as being almost constant during stress relaxation tests as reported in a previous paper $^{(9)}$. 
pendent only on the temperature and material, it follows that $\sigma_{\mathrm{i}}$ must vary during each stress relaxation. In fatigue tests, three-dimensional cell structures are formed at high strain amplitude $\left(\gamma_{\mathrm{p}}>10^{-3}\right)$, wheares dislocation tangles, dense dislocation clusters, heavily jogged screw dislocations and dislocation loops are observed at low strain amplitude $\left(\gamma_{\mathrm{p}}<10^{-3}\right)$. The dislocation structures of the latter are rather unstable ${ }^{(10)}$. As the present fatigue tests belong to the category of low strain amplitude, it seems that $\sigma_{\mathrm{i}}$ changes as the unstable dislocation structure changes during stress relaxation tests and the curves become straight.

The scaling relation did not hold for single crystals, as seen in Fig. 13. As the stress relaxation proceeds, the applied stress decreases continuously after its stagnation for a little while. The initial stagnation of stress may result from the instantaneous immobilization of moving dislocations when the crosshead is stopped. Though the deformation stagnation like this appeared in the early stage of relaxation run of single crystals, it was not observed in polycrystals. The difference may be caused by many obstacles such as grain boundaries, voids produced during sintering and impurities in polycrystals. In other words, these obstacles prevent the dislocations in polycrystals from bowing out, wheares the dislocations in single crystals can bow out easily and react the change in applied stress sensitively. The drastic decrease in $\rho_{\mathrm{m}}$ at the beginning of relaxation run in single crystals can also be predicted from the remarkable change in the activation volume for dislocation movement. The experimental activation volume $V^{*}$ is given by

$$
V^{*}=k T\left(\partial \ln \dot{\gamma}_{\mathrm{p}} / \partial \tau\right)_{T},
$$

where $k$ is the Boltzmann constant and $T$ the absolute temperature. Two regions in Fig. 13 where the deformation stagnates and subsequent continuous stress drop occurs will be designated by (A) and (B), respectively. The activation volumes $V^{*} / b^{3}$ of the regions (A) and (B) were $110-60$ and $35-20$, respectively. They decreased continuously within the ranges, as the plastic strain increased. The difference in the experimental activation volume $V^{*}$ between (A) and (B) can be ascribed to the change in $\rho_{\mathrm{m}}$ in the region of (A). By using the mean dislocation velocity $\bar{v}$ and $\dot{\gamma}_{\mathrm{p}}=\rho_{\mathrm{m}} b \bar{v}$, eq. (15) leads to

$$
V^{*}=k T\left(\partial \ln \rho_{\mathrm{m}} / \partial \tau\right)_{T}+k T(\partial \ln \bar{v} / \partial \tau)_{T}
$$

The right hand second term is the true activation volume. It seems that the first term in the region (A) is considerably large compared with that in the region (B), because the experimental activation volume in the region (B) agrees well with that ${ }^{(11)}$ estimated from both of stress relaxation and strain rate cycling tests with the same (101)[111] single crystals.

The activation volume $V^{*}$ is rewritten using $\lambda$ from eqs. (2) and (15) to be

$$
V^{*}=k T / \tau \lambda \text {. }
$$

In the entire (B) region of Fig. 13, the relations of the relaxed stress $\Delta \tau$ vs $\ln \dot{\gamma}_{\mathrm{p}}$ were linear. This implies that $V^{*}$ remains constant in the (B) region and $\lambda$ increases as $\tau$ decreases under the condition as seen in eq. (17), namely the relaxation curves become concave downward. The result that $V^{*}$ remains constant regardless of the decrease in $\tau$ is contrary to the theoretical prediction $^{(12)}$ or the experimental results ${ }^{(13)}$ in which the inverse relation of $V^{*}$ to $\tau^{*}$ holds for the Peierls mechanism. The contradiction may result from the unnegligible change in the right hand first term in eq. (16) which can cause $V^{*}$ to be constant apparently. Therefore, the invalidity of scaling for single crystals is ascribed to the appreciable change in the mobile dislocation density $\rho_{\mathrm{m}}$ (or $\dot{\varepsilon}_{0}$ ) during each stress relaxation.

\section{A phenomenological interpretation of the mechanical equation of state}

The plastic strain rate $\dot{\varepsilon}$ during stress relaxation tests can be expressed by

$$
\dot{\varepsilon}=\dot{\varepsilon}_{0} \exp \left[-\Delta G\left(\sigma^{*}\right) / k T\right],
$$

where $\dot{\varepsilon}_{0}$ is the preexponential term and $\Delta G$ the Gibbs free energy of activation which increases as $\sigma^{*}$ decreases. $\mathrm{Li}^{(14)}$ calculated the average dislocation velocity from the time required for dislocations to journey in sinusoidal internal stress fields and obtained the mean internal stress $\sigma_{i}^{*}$ connected directly to the average dislocation velocity as a function of the maximum internal stress $\sigma_{\mathbf{i}}^{0}$ and the applied stress. 
By applying Li's theory as reported in the previous paper ${ }^{(9)}$, one obtains the following equation.

$$
\sigma^{*}=\sigma-g\left(\sigma_{\mathbf{i}}^{0}, \sigma\right),
$$

where the mean internal stress $\sigma_{i}^{*}$ is designated as $g\left(\sigma_{\mathbf{i}}^{0}, \sigma\right)$ and the maximum internal stress $\sigma_{i}^{0}$ corresponds to the stress below which the deformation stagnates. Substitution of eq. (19) into eq. (18) gives

$$
\dot{\varepsilon}=\dot{\varepsilon}_{0} \exp \left[-\Delta G\left\{\sigma-g\left(\sigma_{\mathrm{i}}^{0}, \sigma\right)\right\} / k T\right] .
$$

Since a stress relaxation curve at a given temperature can be regarded as the relation between $\dot{\varepsilon}$ and $\sigma$ under the condition that $\dot{\varepsilon}_{0}$ and $\sigma_{\dot{i}}^{0}$ remain approximately constant, eq. (20) leads to

$$
f\left[\dot{\varepsilon}, \sigma,\left(\dot{\varepsilon}_{0}, \sigma_{\mathbf{i}}^{0}\right), T\right]=0 .
$$

Thus, the mechanical equation of state holds for an arbitrary set of $\left(\dot{\varepsilon}_{0}, \sigma_{i}^{0}\right)$. Both of $\dot{\varepsilon}_{0}$ and $\sigma_{i}^{0}$ vary among any relaxation curves, because the history prior to stress relaxation differs from each other. If the relation between $\dot{\varepsilon}_{0}$ and $\sigma_{i}^{0}$ is clarified, and if a series of relaxation curves can be superposed on one another to form a master curve, the practical scaling locus can be calculated from the relation between $\dot{\varepsilon}_{0}$ and $\sigma_{\mathbf{i}}^{0 \dagger}$. However, one should note that the present scaling relation is not a necessary condition but a sufficient one for the validity of the mechanical equation of state as eq. (21).

In order to obtain the relation between $\dot{\varepsilon}_{0}$ and $\sigma_{\mathrm{i}}^{0}$, the following four equations are assumed.

$$
\begin{aligned}
& \rho_{T}=\rho_{0}+C \varepsilon^{\beta}, \quad{\text { (Gilbert } \left.\text { et } a l . .^{(15)}\right)}^{\rho_{\mathrm{m}}}=A \varepsilon, \\
& \alpha_{\mathbf{i}}^{0}=\alpha_{1} G b \sqrt{\rho_{T}}
\end{aligned}
$$

and

$$
\dot{\varepsilon}_{0}=\alpha \rho_{\mathrm{m}} \frac{b a L}{1^{*}}\left(\frac{b}{1^{*}} v_{\mathrm{d}}\right)=\rho_{\mathrm{m}} v,
$$

$\dagger$ The geometrical scaling locus is given by the relation between the stress $\sigma_{i}^{0}$ (or the maximum internal stress) and the strain rate $\dot{\varepsilon}_{\mathrm{c}}\left[=\dot{\varepsilon}_{0} \exp \left(-\Delta G_{0} / k T\right)\right]$ for deformation stagnation. Here, $\Delta G_{0}$ is the Gibbs free energy, when $\sigma^{*}$ is zero. Shifting $\ln \dot{\varepsilon}_{\mathrm{c}}$ by $\Delta G_{0} / k T, \ln \dot{\varepsilon}_{\mathrm{c}}$ can be translated into $\ln \dot{\varepsilon}_{0}$, which will be used as an abscissa in Fig. 14. Similarly, the scaling relation such as Fig. 3 can be depicted as a $\log \sigma_{i}^{0}$ vs $\log \dot{\varepsilon}_{0}$ relation. where $C, \mathrm{~A}$ and $\alpha_{1}$ are constants, $G$ the shear modulus, $l^{*}$ the activated length of dislocation, $a \cdot L$ the activation area, $v_{\mathrm{d}}$ the Debye frequency and $v$ the frequency factor. Combination of eqs. (22) to (25) gives

$$
\dot{\varepsilon}_{0}=A v\left[\frac{1}{C}\left\{\left(\frac{\sigma_{\mathrm{i}}^{0}}{\alpha_{1} G b}\right)^{2}-\rho_{0}\right\}\right]^{1 / \beta} .
$$

By putting $A=10^{10} / \mathrm{cm}^{2}, C=8 \times 10^{10} / \mathrm{cm}^{2(15)}$, $\left.\rho_{0}=3 \times 10^{8} / \mathrm{cm}^{2(15)}, \alpha_{1}=0.72^{(16)}, v=10^{-3} \mathrm{~cm}^{2}\right)$ $\mathrm{s}^{(17)}$ into eq. (26), the relation between $\dot{\varepsilon}_{0}$ and $\sigma_{i}^{0}$ can be calculated as a function of $\beta$ and shown in Fig. 14. A good linear relationship was obtained within the experimentally measured range indicated by the dotted line. When all of $\varepsilon, \rho_{\mathrm{m}}$ and $\dot{\varepsilon}$ in eqs. (23) to (25) are zero, the internal stress is designated by $\sigma_{\mathbf{i}_{\mathbf{x}}}^{0}$. The values of $\beta$ in the figure were selected so as to give the temperature dependence of $m(=2.3-8.6)$ for pure molybdenum wires shown in Fig. 11. At $292 \mathrm{~K}, \beta$ was chosen to be 0.2 , which was fairly small compared with that (1.0) obtained from the electron microscopic observation at room temperature ${ }^{(15)}$. The discrepancy may result from the difference in the method of measurement. In other words, the latter observation gives the direct relation between $\sigma_{T}$ and $\varepsilon$, whereas the strain dependence of $\rho_{\mathrm{m}}$ is assumed to be eq. (23), and $\beta$ is selected to give the scaling relation between $\rho_{T}$ (or $\sigma_{\mathrm{i}}^{0}$ ) and $\rho_{\mathrm{m}}$ (or $\dot{\varepsilon}_{0}$ ) as seen eq. (26) in the present study. As mentioned above, such factors as (1)-(A) and (2) in Fig. 1 and (4) grain size had no effect on $m$. This means that $\beta$ does not depend on such factors. On the other hand, $m$ depended on impurities and the temperature. The dependence is presented as a function of $\beta$ in Fig. 15 in which the temperature is indicated only for pure molybdenum wires. It is known from the figure that $\beta$ has a tendency to increase as the temperature and the amount of impurities increase. If the change in $\rho_{\mathrm{m}}$ is described instead of $\beta$ under the condition that $\sigma_{\mathbf{i}}^{0}$ (or $\rho_{T}$ ) is constant, it follows from eqs. (25) and (26) that $\rho_{\mathrm{m}}$ increases as the temperature and the amount of impurities increase. Accordingly, it is concluded that there is no effect of such factors as (1)-(A) cyclic loading, (2) imposed strain rate and (4) grain size on $\rho_{\mathrm{m}}$.

Thus, the scaling locus of stress relaxation 


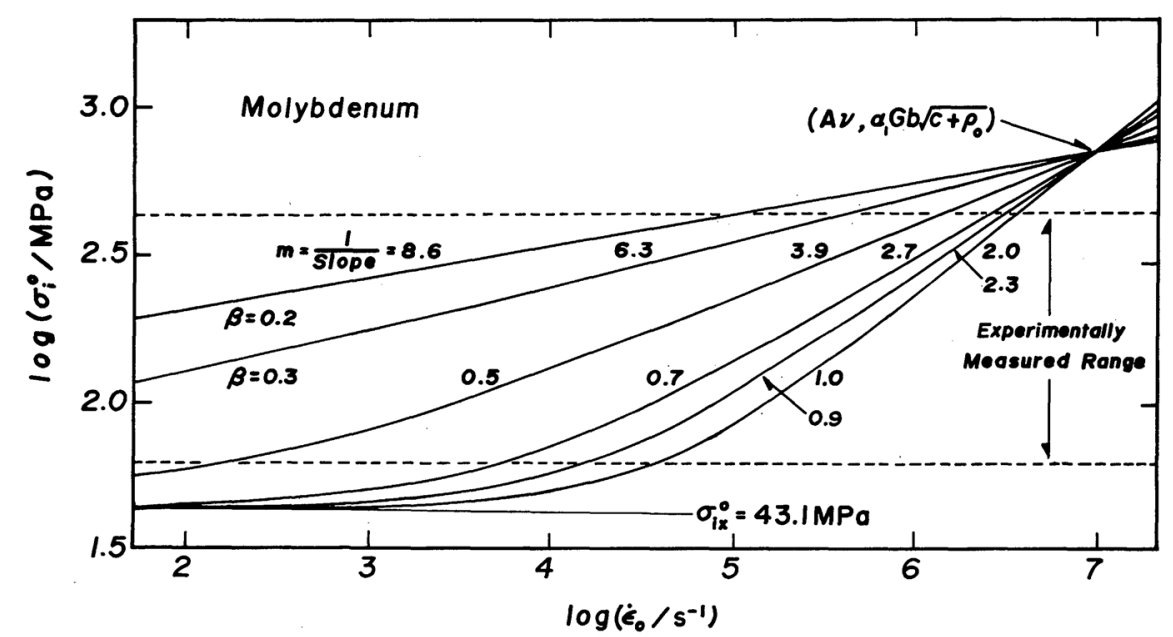

Fig. 14 Various scaling loci calculated from eq. (26) which expresses the relation between the preexponential term $\dot{\varepsilon}_{0}$ and the maximum internal stress $\sigma_{i}^{0}$.

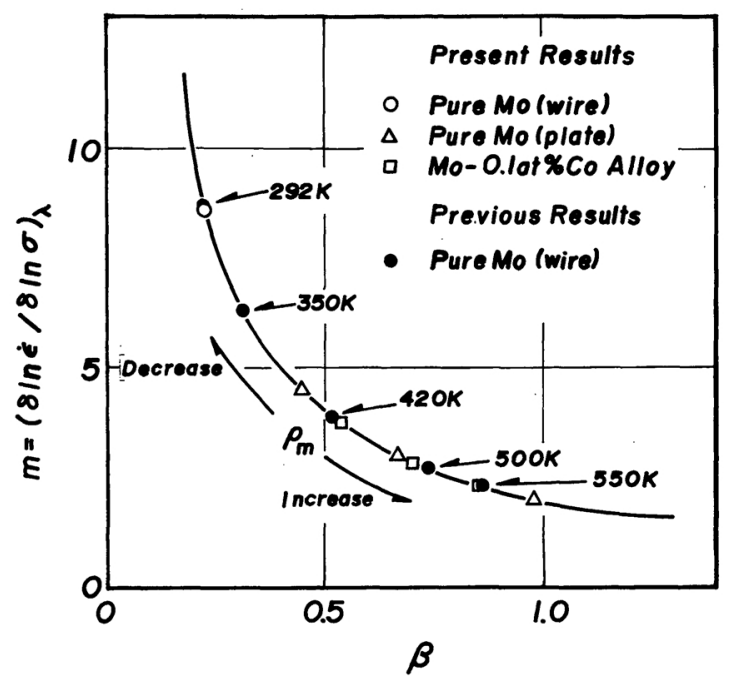

Fig. 15 Variation of $m$ with $\beta$ as a strain exponent. The increase in $\beta$ or the decrease in $m$ causes the mobile dislocation density $\rho_{\mathrm{m}}$ to increase.

curves can be explained by the relation between $\dot{\varepsilon}_{0}$ and $\sigma_{\mathbf{i}}^{0}$. If the effect of various factors on $\beta$ and the relation between $\Delta G$ and stress in eq. (20) are further clarified and the scaling is possible, the mechanical equation of state as eq. (21) holds for the arbitrary set of $(\dot{\varepsilon}, \sigma, T)$, and the parameters concerning the dislocation structure such as $\rho_{\mathrm{m}}, \rho_{T}$ and $\varepsilon$ can be simultaneously determined.

\section{Conclusion}

Stress relaxation curves, obtained for polycrystalline molybdenum by a monotonic loading mode, were concave upward, and a unique master curve was formed by scaling. The scaling parameter $m$ was then obtained as a reciprocal of the slope on scaling. The effect of such various factors as (1) cyclic loading, (2) imposed strain rate (shown in Fig. 1), (3) impurities and (4) grain size on $m$ were investigated over the temperature range from 292 to $550 \mathrm{~K}$. The experimental results are summarized as follows.

(1) Such factors as (1)-(A) the stress amplitude and the cycles of cyclic loading and (2) the imposed strain rate had no effect on $m$ and the shape of stress relaxation curves. Similarly, the effect of (4) grain size on $m$ was not observed.

(2) In the fatigue test shown in Fig. 1(1)(B), the scaling relation did not hold, because the shape of the stress relaxation curves became straight and their slopes were defferent. In single crystals, the shape of the curves became concave downward and the scaling was again invalid.

(3) Such factors as (3) impurities and temperature varied the shape of relaxation curves as well as the scaling parameter $m$.

The physical meaning of scaling was discussed on the basis of these experimental re- 
sults. Consequently, the scaling locus can be described by the relation between the preexponential term $\dot{\varepsilon}_{0}$ (or $\rho_{\mathrm{m}}$ ) and the maximum internal stress $\sigma_{\mathrm{i}}^{0}$ as given in eq. (26). Moreover, it is concluded that such a mechanical equation of state as eq. (21) is valid for an arbitrary set of $(\dot{\varepsilon}, \sigma, T)$ under the present condition except for the fatigue tests and single crystals.

\section{Acknowledgements}

The authors wish to acknowledge $\mathrm{Mr}$. $\mathrm{K}$. Senba of Nippon Tungsten Co., Ltd. for providing molybdenum ingots, and Messrs. T. Terada and S. Muramoto for their experimental assistance. Professor K. Kitajima of Kyushu University offered convenience to us for the provision of molybdenum single crystals.

\section{REFERENCES}

(1) E. W. Hart, C. Y. Li, H. Yamada and G. L. Wire: Constitutive Equations in Plasticity, ed. by A. S. Argon, MIT Press, Cambridge, (1975), p. 149.

(2) E. W. Hart and H. D. Solomon: Acta Met., 21 (1973), 295.

(3) H. Yamada and C. Y. Li: Acta Met., 22 (1974), 249.
(4) G. L. Wire, H. Yamada and C. Y. Li: Acta Met. 22 (1974), 505.

(5) E. W. Hart: Rate Processes in Plastic Deformation of Materials, ed. by J. C. M. Li and A. K. Mukherjee, ASM (1975), p. 284.

(6) J. J. Gilman: Aust. J. Phys., 13 (1960), 327.

(7) K. Tanoue and H. Matsuda: Trans. JIM, 23 (1982), 234.

(8) K. Abe, H. Yoshinaga and S. Morozumi : J. Japan Inst. Metals, 40 (1976), 393 (in Japanese).

(9) K. Tanoue and H. Matsuda: Trans. JIM, 23 (1982), 727.

(10) J. C. Grosskreutz and M. Mughrabi: Constitutive Equations in Plasticity, ed. by A. S. Argon, MIT Press, Cambridge (1975), p. 251.

(11) F. Guiu: Phys. Status Solidi, 19 (1967), 339.

(12) P. Guyot and J. E. Dorn: Canad. J. Phys., 45 (1967), 983.

(13) H. Conrad and W. Hayes: Trans. ASM, 56 (1963), 249.

(14) J. C. M. Li: Dislocation Dynamics, ed. by A. R. Rosenfield et al., McGraw-Hill, New York, (1968), p. 87.

(15) A. Gilbert, B. A. Wilcox and G. T. Hahn: Phil. Mag., 12 (1965), 649.

(16) S. Karashima, H. Oikawa and T. Igarashi: J. Japan Inst. Metals, 34 (1970), 389 (in Japanese).

(17) K. E. Amin, K. L. Murty and J. E. Dorn: J. Appl. Phys., 44 (1973), 615. 